\title{
Short-term forecasting of the COVID-19 pandemic using Google Trends data: Evidence from 158 countries
}

\begin{abstract}
The ability of Google Trends data to forecast the number of new daily cases and deaths of COVID-19 is examined using a dataset of 158 countries. The analysis includes the computations of lag correlations between confirmed cases and Google data, Granger causality tests, and an out-of-sample forecasting exercise with 18 competing models with a forecast horizon of 14 days ahead. This evidence shows that Google-augmented models outperform the competing models for most of the countries. This is significant because Google data can complement epidemiological models during difficult times like the ongoing COVID-19 pandemic, when official statistics maybe not fully reliable and/or published with a delay. Moreover, real-time tracking with online-data is one of the instruments that can be used to keep the situation under control when national lockdowns are lifted and economies gradually reopen.
\end{abstract}

Keywords: COVID-19; Google Trends; VAR; ARIMA; ARIMA-X; ETS; LASSO; SIR model. JEL classification: C22; C32; C51; C53; G17; I18; I19.

\section{Introduction}

T he SARS-CoV-2 virus was initially detected in Wuhan (China) in December 2019 (Li et al., 2020a), and then rapidly spread to all Chinese provinces and the rest of the world in the subsequent months. This chain of events led the World Health Organization (WHO, 2020) to declare a pandemic for the Coronavirus disease (COVID-19) on the 11/03/2020. At the end of May 2020, confirmed coronavirus cases worldwide surpassed 4 million and there were at least 300000 deaths in more than 180 countries (ECDC, 2020).

A large literature investigated how internet search data from search engines and data from traditional surveillance systems can be used to compute real-time and short term forecasts of several diseases, see Ginsberg et al. (2009), Broniatowski et al. (2013), Yang et al. (2015), and Santillana et al. (2015): these approaches could predict the dynamics of disease epidemics several days or weeks in advance. Besides, there is a small but quickly increasing literature that examines how internet search data can be used to predict the COVID-19 pandemic, see for example Li et al. (2020b) and Ayyoubzadeh et al. (2020). Moreover, to the best of our knowledge, neither formal Granger causality testing was computed to determine whether Google search data is useful for forecasting COVID-19 cases, nor large scale out-of-sample forecasting analysis was performed. The possibility to predict the dynamics of a dangerous pandemic is of fundamental

1 Fantazzini, Dean - Moscow School of Economics, Moscow State University, Moscow; fantazzini@mse-msu.ru. This paper is dedicated to the memory of the author's mother Maria Pirazzini, who passed away on 16/07/2020. 
importance for policymakers and health officials to prevent its further spread and to evaluate the success of their policy actions to contain the disease.

In this study, we evaluated the ability of Google search data to forecast the number of new daily cases and deaths of COVID-19 using data for 158 countries and a set of 18 forecasting models.

The first contribution of this paper is an evaluation of the contribution of online search queries to the modelling of the new daily cases of COVID-19 for 158 countries, using lag correlations between confirmed cases and Google data, as well as different types of Granger causality tests. To our knowledge, this analysis has not been done elsewhere. The second contribution is an out-of-sample forecasting exercise with 18 competing models with a forecast horizon of 14 days ahead for all countries, with and without Google data. The third contribution of the paper is a robustness check to measure the accuracy of the models' forecasts when forecasting the number of new daily deaths instead of cases.

The rest of this paper is organized as follows. Section 2 briefly reviews the literature devoted to forecasting infectious diseases with Google Trends and online data, while the methods proposed for forecasting the new daily cases and deaths of COVID-19 are discussed in Section 3. The empirical results are reported in Section 4, while a robustness check is discussed in Section 5. Section 6 briefly concludes.

\section{Literature review}

Several authors examined the predictive power of online data to forecast the temporal dynamics of different diseases. They found that these data can offer significant improvements with respect to traditional models: see, just to name a few, Ginsberg et al. (2009), Wilson et al. (2009), Seifter et al. (2010), Valdivia and Monge-Corella (2010), Zhou and Feng (2011), Yin and Ho (2012), Ayers et al. (2013), Yuan et al. (2013), Dugas et al. (2013), Majumder et al. (2016), Shin et al. (2016), Marques-Toledo et al. (2017), Teng et al. (2017), Ho et al. (2018), Gianfredi et al. (2018), Santangelo et al. (2019), Li et al. (2020b).

Milinovich et al. (2014) provides one of the first and largest reviews of this literature and explains the main reasons behind the predictive power of online data. The idea is quite simple: people suspecting an illness tend to search online for information about the symptoms and, if possible, how they can self-medicate. The last reason is particularly important in those countries where basic universal health care and/or paid sick leave are not available.

Traditional epidemiologic models to forecast infectious diseases may lack flexibility, be computationally demanding, or require data that are not available in real-time, thus strongly reducing their practical utility: see, for example, Longini et al. (1986), Hall et al. (2007), Birrell et al. (2011), Boyle et al. (2011), and Dugas et al. (2013).

Instead, internet-based surveillance systems are generally easy to compute and they are economically affordable even for poor countries. Moreover, they can be used together with traditional surveillance approaches. However, internet-based surveillance systems have also important limitations: they can be strongly influenced by the mass media, which can push frightened people to search for additional information online, thus misrepresenting the real situation on the ground. This is what happened during the 2012-2013 influenza season, when Google Flu Trends (GFT) overestimated the prevalence of influenza by more than fifty percent, see Lazer et al. (2014) for more details. Therefore, internet-based systems should not be viewed as an alternative to tradi- 
tional surveillance systems, but rather an extension. Moreover, they can improve the ability to forecast the temporal dynamics of dangerous illnesses when official statistics are not available or available with a delay, and when official statistics may not be fully reliable.

We remark that already in the middle of 2020 there is a very large literature devoted to forecasting techniques for the COVID-19 disease. Shinde et al. (2020) provide a state-of-the-art survey of this literature by reviewing 47 papers, including both published papers and pre-prints without peer-review. They categorize these papers into 4 categories: (a) Big data; (b) Social media/other communication media data; (c) Stochastic theory/mathematical models; (d) Data science/Machine learning techniques. They examined each paper focusing on the specific countries used for the analysis, the various statistical, analytical, mathematical and medical (symptomatic and asymptomatic) parameters taken into consideration in the paper, and the main outcomes of the paper. Interestingly, despite several useful findings, Shinde et al. (2020) highlight that there are still issues and challenges which need to be addressed. The first and most important issue is the excessive reliance on China's dataset for model testing and forecasting, and the need to consider different datasets to verify ".... whether the same mathematical or prediction model is also suitable to predict the spread and reproduction number for all the countries across the globe" (Shinde et al., 2020, p. 197). This was by far the most important driver behind the writing of this paper, and which guided our research effort. Moreover, other challenges identified by Shinde et al. (2020, p. 197) like longer incubation period, lack of proper data, over-fitting of the data, overly clean data and model complexity influenced our choices in terms of model selection and data sources: these issues will be discussed in more details in section 3 and 4.1, respectively.

Gencoglu and Gruber (2020) were the first to try to discover and quantify causal relationships between the number of infections and deaths and online sentiment as measured by Twitter activity. This is important because distinguishing epidemiological events that correlate with public attention from epidemiological events that cause public attention is fundamental when preparing public health policies. However, Gencoglu and Gruber (2020) did not attempt to model temporal causal relationships: this was one of the main reason why we decided to consider different types of Granger causality tests in our analysis, as discussed below in section 3.1.

Finally, we note that there is a strand of the literature that extends classical epidemiologic models to study the interaction between economic decisions and epidemics, see Eichenbaum et al. $(2020 \mathrm{a}, \mathrm{b}, \mathrm{c})$ for more details. These works abstract from many important real-world complications to focus on the basic economic forces at work during an epidemic, and to show that "there is an inevitable trade-off between the severity of the short-run recession caused by the epidemic and the health consequences of that epidemic" (Eichenbaum et al., 2020a, p. 28). Given that these models are more intended for policy making rather than forecasting, we did not consider them for this work.

\section{Methodology}

The goal of this paper is to verify whether Google Trends data is useful for forecasting new daily cases/deaths of COVID-19. To this end, we will compute Granger causality tests and a large-scale out-of-sample forecasting analysis. Before presenting the results of the empirical analysis, we briefly review the theory of Granger causality testing and the forecasting models that we will use to predict the daily cases and deaths of COVID-19. 


\subsection{Granger Causality}

\subsubsection{Brief review of the theory}

Wiener (1956) was the first to propose the idea that, if the prediction of one time series can be improved by using the information provided by a second time series, then the latter is said to have a causal influence on the first. Granger $(1969,1980)$ formalized this idea in the case of linear regression models. In general, if a variable (or a set of variables) $X$ can improve forecasting another variable (or a set of variables) $Y$, then we say that $X$ Granger-causes $Y$. Otherwise, $X$ fails to Granger-cause $Y$.

Let's consider a general setting for a $\operatorname{VAR}(p)$ process with $n$ variables,

$$
Y_{t}=\alpha+\Phi_{1} Y_{t-1}+\Phi_{2} Y_{t-2}+\ldots+\Phi_{p} Y_{t-p}+\varepsilon_{t},
$$

where $Y_{t}, \alpha$ and $\varepsilon_{t}$ are $n$-dimensional vectors; $\Phi_{i}$ is an $n \times n$ matrix of autoregressive parameters for lag $i$.

The $\operatorname{VAR}(p)$ process can be written more compactly as,

$$
Y=B Z+U,
$$

where $Y=\left(Y_{1}, \ldots, Y_{T}\right)$ is a $(n \times T)$ matrix; $B=\left(\alpha, \Phi_{1}, \ldots, \Phi_{p}\right)$ is a $(n \times(1+n p))$ matrix; $Z=\left(Z_{0}, \ldots, Z_{T-1}\right)$ is a $((1+n p) \times T)$ matrix with $Z_{t}=\left[1 Y_{t} \ldots Y_{t-p+1}\right]^{\prime}$ a $(1+n p)$ vector; $U=\left(\varepsilon_{1}, \ldots, \varepsilon_{T}\right)$ is a $(n \times T)$ matrix of error terms.

If we want to test for Granger-causality, we need to test a set of zero constraints on the coefficients: for example, the $k$-th element of $Y_{t}$ does not Granger-cause the $j$-th element of $Y_{t}$ if the row $j$, column $k$ element in $\Phi_{i}$ equals zero for $i=1, \ldots, p$. More generally, if we define $\beta=\operatorname{vec}(B)$ as a $\left(n^{2} p+n\right)$ vector with vec representing the column-stacking operator, the null hypothesis of no Granger-causality can be expressed as

$$
\mathbf{H}_{\mathbf{0}}: C \beta=0 \text { vs } \mathbf{H}_{\mathbf{1}}: C \beta \neq 0,
$$

where $C$ is an $\left(N \times\left(n^{2} p+n\right)\right)$ matrix; 0 is an $(N \times 1)$ vector of zeroes; $N$ is the total number of coefficients restricted to zero.

It is possible to show that the Wald statistic defined by

$$
(C \hat{\beta})^{\prime}\left[C\left((Z Z)^{-1} \otimes \hat{\Sigma}_{U}\right) C\right]^{-1}(C \hat{\beta})
$$

has an asymptotic $\chi^{2}$ distribution with $N$ degrees of freedom, where $\hat{\beta}$ is the vector of estimated parameters, while $\hat{\Sigma}_{U}$ is the estimated covariance matrix of the residuals, see Lütkepohl (2005, section 3.6.1) for a proof.

\subsubsection{Dealing with non-stationary data: The Toda-Yamamoto approach}

It is well known that the use of non-stationary data can deliver spurious causality results, see Granger and Newbold (1974), Phillips (1986), Park and Phillips (1989), Stock and Watson (1989), and Sims et al. (1990). Moreover, Sims et al. (1990) showed that the asymptotic distribution theory cannot be applied for testing causality even in the case when the variables are cointegrated. 
Toda and Yamamoto (1995) introduced a Wald test statistic that asymptotically has a chisquare distribution even if the processes may be integrated or cointegrated of arbitrary order. Their approach requires, first, to determine the optimal VAR lag length $p$ for the variables in levels using information criteria. Secondly, a $(p+d)$ th-order VAR is estimated, where $d$ is the maximum order of integration for the set of variables. Finally, Toda and Yamamoto (1995) showed that we can test linear or nonlinear restrictions on the first $p$ coefficient matrices using standard asymptotic theory, while the coefficient matrices of the last $d$ lagged vectors can be disregarded. Therefore, after a $\operatorname{VAR}(p+d)$ model is estimated, the vector of the stacked estimated parameters can be modified as $\hat{\beta}=\operatorname{vec}\left(\alpha, \hat{\Phi}_{1}, \ldots, \hat{\Phi}_{p}, 0_{n \times n d}\right)$, where $0_{n \times n d}$ denotes a zero matrix with $n$ rows and $n d$ columns, and equation (1) can then be used. Note that there are special cases when the extra lag (s) are not necessary to obtain the asymptotic $\chi^{2}$-distribution of the Wald test for Granger-causality, see Lütkepohl (2005, section 7.6.3) for more details.

\subsection{Forecasting methods}

We will perform an out-of-sample forecasting analysis for each country, to predict the number of daily new cases and deaths using three classes of models: time series models, Googleaugmented time series models and epidemiologic models. A brief description of each model is reported below.

\subsubsection{Time series models}

Auto-Regressive Integrated Moving Average (ARIMA) models represent an important benchmark in time series analysis and we refer the interested reader to Hamilton (1994) for a detailed discussion at the textbook level. For sake of generality and interest (see section 4.1 below for more details), we considered models with the variables in levels, log-levels, first differences and log-returns. The optimal number of lags for the auto-regressive and moving average terms were chosen by minimizing the Akaike information criteria (AIC). ARIMA models are important benchmarks in the famous forecasting competitions known as Makridakis Competitions (or M-Competitions ${ }^{2}$ ), see Makridakis and Hibon (2000) and Makridakis et al. (2020) for more details.

Exponential smoothing methods have a long tradition in the statistical literature: recently, they have witnessed a revival due to their inclusion in a general dynamic nonlinear framework, which allows their implementation into state space form with several extensions, see Hyndman et al. (2002) and Hyndman et al. (2008). This more general class of models is known as ETS (Error-Trend-Seasonal or ExponenTial Smoothing), and it includes standard exponential smoothing models, like the Holt and Holt-Winters additive and multiplicative methods. The general structure of this framework is to decompose a time series $Y$ into three components: a trend $(T)$ which represents the long-term component of $Y$, a seasonal pattern $(S)$, and an error term $(E)$. These components can enter the model specification as additive terms (for example $Y=T+S+E$ ), multiplicative (for example $Y=T \cdot S \cdot E$ ) or both (for example, $Y=T \cdot S+E$ ). Moreover, the trend component can be decomposed into a level term $(l)$ and a growth term $(b)$ which can be "damped" by using an additional parameter $0<\phi<1$, so that five different trend types are possible: 1 ) none: $T_{h}=l ; 2$ ) additive: $\left.T_{h}=l+b h ; 3\right)$ additive damped: $T_{h}=l+b \phi_{h}$;

2 https://en.wikipedia.org/wiki/Makridakis_Competitions. 
4) multiplicative: $\left.T_{h}=l \cdot b^{h} ; 5\right)$ multiplicative damped: $T_{h}=l \cdot b^{\phi_{h}}$, where $T_{h}$ is the trend forecast $h$ periods out, while $\phi_{h}=\sum_{s=1}^{h} \phi^{s}$. Therefore, we can have $30=5 \cdot 3 \cdot 2$ possible ETS models, see Hyndman et al. (2008) for more details.

Assuming a general state vector $x_{t}=\left[l_{t}, b_{t}, s_{t}, s_{t-1}, \ldots, s_{t-m}\right]$ where $l_{t}, b_{t}$ are the previous trends components and $s_{t}$ the seasonal terms, a state-space representation with a common error term of an exponential smoothing model can be written as follows:

$$
y_{t}=h\left(x_{t-1}\right)+k\left(x_{t-1}\right) \varepsilon_{t}, \quad x_{t}=f\left(x_{t-1}\right)+g\left(x_{t-1}\right) \varepsilon_{t},
$$

where $h$ and $k$ are continuous scalar functions; $f$ and $g$ are functions with continuous derivatives; $\varepsilon_{t} \sim \operatorname{NID}\left(0, \sigma^{2}\right)$. The equation for $y_{t}$ shows how the various state variable components $\left(l_{t-1}, b_{t-1}, s_{t-1}, \ldots\right)$ are combined to express $y_{t}$ as a function of a smoothed forecast $\hat{y}_{t}=h\left(x_{t-1}\right)$ with an error term $\varepsilon_{t}$, while the equation for $x_{t}$ show how these state variable components are updated. The detailed equations for all the 30 possible ETS models are reported in Hyndman et al. (2008 Tables 2.2 and 2.3, p. 21-22).

ETS models are estimated by maximizing the likelihood function with multivariate Gaussian innovations. Hyndman et al. (2008) showed that twice the negative logarithm of the likelihood function conditional on the model parameters $\theta$ and the initial states $x_{0}=\left(l_{0}, b_{0}, s_{0}, s_{t-1}, \ldots\right)$ and without constants, is given by:

$$
L^{*}\left(\theta ; x_{0}\right)=n \ln \left(\sum_{t=1}^{n} \frac{\varepsilon^{2}}{k^{2}\left(x_{t-1}\right)}\right)+2 \sum_{t=1}^{n} \ln \left|k\left(x_{t-1}\right)\right| .
$$

The parameters $\theta$ and the initial states $x_{0}$ are then estimated by minimizing $L^{*}\left(\theta ; x_{0}\right)$. The model selection is then performed using information criteria. Like ARIMA models, the ETS model is also an important benchmark in the famous M-competitions. Both ARIMA and ETS models provide good forecasts in the short term, but the quality of these forecasts quickly decreases with an increasing forecast horizon.

\subsubsection{Google-augmented time series models}

The easiest way to include Google search data in a time series model is probably by using an ARIMA model with eXogenous variables (ARIMA-X), see Hyndman and Athanasopoulos (2018) for more details. More specifically, we employed a simple ARIMA model augmented with the Google search data for the topic 'pneumonia' lagged by 14 days. This choice was based on two considerations: first, the WHO (2020) officially states that "the time between exposure to COVID-19 and the moment when symptoms start is commonly around five to six days but can range from 1-14 days". Second, Li et al. (2020b) showed that the daily new COVID-19 cases in China lag online search data for the topics 'coronavirus' and 'pneumonia' by 8-14 days, depending on the social platform used. We chose only the searches for the topic 'pneumonia' because they are less affected by news-only related searches. Even though such a simple ARIMA-X model is definitely biased, this parsimonious model can nevertheless be of interest for forecasting purposes. Moreover, the capacity of Google data to summarize a wealth of information should not be underestimated, as shown by Fantazzini (2014) and Fantazzini and Toktamysova (2015). Similarly to the previous ARIMA models, we considered four ARIMA-X models with the variables in levels, log-levels, first 
differences and log-returns. We remind the reader that Google Trends data represents the number of searches in Google for a topic or a keyword divided by the total amount of searches for the same period and region, and standardized between 0 and 100. A detailed description of how this variable is computed and some examples are reported in the Appendix A in the Supplementary Materials ${ }^{3}$.

A more general framework is a trivariate $\operatorname{VAR}(p)$ model,

$$
Y_{t}=v+\sum_{i=1}^{p} \Phi_{i} Y_{t-i}+u_{t}, \quad u_{t} \sim W N\left(0, \Sigma_{u}\right),
$$

where $Y_{t}$ is a $(3 \times 1)$-vector containing the daily new cases of COVID-19 on day $t$, the daily Google search data for the topics 'coronavirus' and 'pneumonia' filtered using the 'Health' category to avoid news-related searches; $v$ is an intercept vector; $\Phi_{i}$ are the usual coefficient matrices with $i=1, \ldots, p$. Similarly to ARIMA models, we considered four possible $\operatorname{VAR}(p)$ with the variables in levels, log-levels, first differences and log-returns, while the optimal lags were again selected using the Akaike information criteria. Given the small sample size at our disposal, higherdimensional system were not considered because $\operatorname{VAR}(p)$ models suffer from the curse of dimensionality (the number of the parameters is equal to $k+p k^{2}$, where $k$ is the number of time series). Similarly, we excluded cointegrated-VAR models due to their computational problems with small samples and noisy Google data, see Fantazzini and Toktamysova (2015, section 4.4), Fantazzini (2019, section 7.6) and references therein for more details.

Unfortunately, even a simple trivariate $\operatorname{VAR}(p)$ can have a wealth of parameters: for example, if $p=14,129$ parameters need to be estimated, which may be very difficult if not impossible to do, depending on the sample size. It is for this reason, that the Hierarchical Vector Autoregression (HVAR) model estimated with the Least Absolute Shrinkage and Selection Operator (LASSO) proposed by Nicholson et al. (2017) and Nicholson et al. (2018) is an ideal approach in the case of a large number of parameters and a small dataset. Let us consider again the previous $\operatorname{VAR}(p)$ process,

$$
Y_{t}=v+\sum_{i=1}^{p} \Phi_{i} Y_{t-i}+u_{t}, \quad u_{t} \sim W N\left(0, \Sigma_{u}\right),
$$

where $Y_{t}$ is a $(3 \times 1)$-vector containing the daily new cases of COVID-19, and the daily Google data for the topics 'coronavirus' and 'pneumonia'. The HVAR approach proposed by Nicholson et al. (2018) adds structured convex penalties to the least squares VAR problem to induce sparsity and a low maximum lag order, so that the optimization problem is given by

$$
\min _{v, \Phi} \sum_{t=1}^{T}\left\|Y_{t}-v-\sum_{i=1}^{p} \Phi_{p} Y_{t-i}\right\|_{F}^{2}+\lambda\left(\mathcal{P}_{Y}(\Phi)\right),
$$

where $\|A\|_{F}$ denotes the Frobenius norm of matrix $A$ (that is, the elementwise 2-norm); $\lambda \geq 0$ is a penalty parameter; $\mathcal{P}_{Y}(\Phi)$ is the group penalty structure on the endogenous coefficient matrices.

We used the elementwise penalty function,

$$
\mathcal{P}_{Y}(\Phi)=\sum_{i=1}^{3} \sum_{j=1}^{3} \sum_{l=1}^{p}\left\|\Phi_{i j, l}\right\|_{2},
$$

3 The supplementary materials can be found on the author's website: https://drive.google.com/file/d/1rCxg_H8wCxYjfMdlAC1WbcNqEhhvt7-R/view?usp=sharing. 
which is the most general structure, allowing every variable in every equation to have its own maximum lag, so that there can be $3^{2}=9$ possible lag orders. The penalty parameter $\lambda$ was estimated by sequential cross-validation, see Nicholson et al. $(2018,2019)$ for the full details. We considered the HVAR model with the variables in levels, log-levels, first differences and log-returns. An example of sparsity pattern with a trivariate HVAR(5) model computed with the elementwise penalty function is reported in Fig. 1.

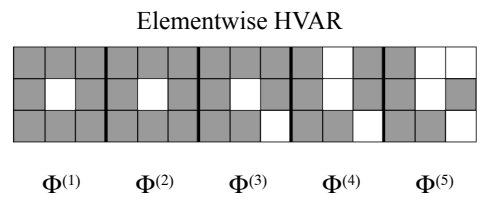

Fig. 1. Example of a trivariate HVAR(5) sparsity pattern, computed with the elementwise penalty function.

Active coefficients are shaded, whereas white cells denote coefficients set to zero

The HVAR model is a special case of a multivariate penalized least squares optimization problem, which can be solved using iterative nonsmooth convex optimization, and which has been recently implemented in the R package BigVAR by Nicholson et al. (2019).

\subsubsection{Epidemiologic models}

The SIR (Susceptible, Infected, Recovered) compartmental epidemic model, was originally proposed by Kermack and McKendrick (1927). Despite its relatively simple assumptions, it is still nowadays one of the main benchmark models in epidemiology, see Brauer and CastilloChavez (2012, chapter 9) for a discussion at the textbook level.

The SIR model assumes that the population is divided into three compartments, where susceptible is a group of people who can be infected, infectious represents the infected people who can transmit the infection to susceptible people but can recover from it, while recovered represents the people who got immunity and cannot be infected again. The SIR model describes the number of people in each compartment using a set of ordinary differential equations:

$$
\frac{d S}{d t}=-\frac{\beta I S}{N}, \quad \frac{d I}{d t}=\frac{\beta I S}{N}-\gamma I, \quad \frac{d R}{d t}=\gamma I,
$$

where $\beta$ models how quickly the disease can be transmitted and it is given by the probability of contact multiplied by the probability of disease transmission, while $\gamma$ models how quickly people recover from the disease.

Note that $N=S(t)+I(t)+R(t)$ represents the total population and it is a constant. The ratio $R_{0}=\beta / \gamma$ is known as the basic reproduction number and it represents the average number of new infected people from a single infected person. The interpretation of this number is easier if we consider that $1 / \gamma$ represents the average time needed to recover from the disease, while $1 / \beta$ is the average time between contacts.

The SIRD (Susceptible, Infectious, Recovered, Deceased) model adds a fourth compartment to model the dynamics of deceased people:

$$
\frac{d S}{d t}=-\frac{\beta I S}{N}, \quad \frac{d I}{d t}=\frac{\beta I S}{N}-\gamma I-\mu I, \quad \frac{d R}{d t}=\gamma I, \quad \frac{d D}{d t}=\mu I,
$$


where $\mu$ is the mortality rate. Anastassopoulou et al. (2020) were the first to use the SIRD model with COVID-19 data. Even though several variants of the SIR model have been proposed, they require additional information that is not publicly available or available with great delay. Moreover, the level of numerical complexity is much higher and this may strongly penalize the efficiency of the model estimates: this will be evident when we will compare the SIR and the SIRD models in section 5. I refer the interested reader to Soetaert et al. (2010a,b) and Brauer and CastilloChavez (2012) for more details about the estimation of systems of ordinary differential equations.

\section{Empirical analysis}

\subsection{Data}

The daily numbers of COVID-19 confirmed cases for over 200 countries were downloaded from the website of the European Centre for Disease Prevention and Control (ECDC), which is an agency of the European Union. To the author's knowledge, this is probably the best COVID-19 dataset currently available. The detailed procedure explaining how the data are collected is reported below for ease of reference:

"Since the beginning of the coronavirus pandemic, ECDC's Epidemic Intelligence team has been collecting on daily basis the number of COVID-19 cases and deaths, based on reports from health authorities worldwide. To insure the accuracy and reliability of the data, this process is being constantly refined. This helps to monitor and interpret the dynamics of the COVID-19 pandemic not only in the European Union (EU), the European Economic Area (EEA), but also worldwide. Every day between 6.00 and 10.00 CET, a team of epidemiologists screens up to 500 relevant sources to collect the latest figures. The data screening is followed by ECDC's standard epidemic intelligence process for which every single data entry is validated and documented in an ECDC database. An extract of this database, complete with up-to-date figures and data visualisations, is then shared on the ECDC website, ensuring a maximum level of transparency" 4 .

Additional comments about this dataset and other COVID-19 datasets can be found in Alamo et al. (2020). We want to remark that only countries that had at least 100 confirmed cases at the time of writing this paper were considered (that is, in May 2020).

Following Li et al. (2020b), we also downloaded the daily data related to the two specific search terms 'coronavirus' and 'pneumonia' from Google Trends, using the time range 01/01/2020-13/05/20. However, our Google dataset differs from Li et al. (2020b) in two aspects. First, we downloaded the data relative to the topics in the place of the simple keywords: search data for topics are cleaned automatically by Google of all searches that are not related to the specific chosen topic. Second, only topics data from the 'Health' category were considered, to avoid all news-related searches. These two filters were used to reduce the noise in the data and improve their predictive ability. Note that the use of Google topics solves automatically the problem of translating the keywords into local languages.

We remark that the issue of weekly seasonality, which is present in mild form both in daily COVID data and in Google searches, was not dealt directly because the estimation of several models would have become very difficult (if not impossible) if additional parameters were added to deal with seasonality. However, we tried to deal with it at least partially in two ways: first, we considered the ETS model that can model seasonality by construction. Had seasonality been an important

4 https://data.europa.eu/euodp/en/data/dataset/covid-19-coronavirus-data. 
factor, ETS models would have out-performed the competitors, but this was not the case. Secondly, Google search data showed a very mild seasonality (people searched more on the weekends than in working days), so that regressing the number of daily cases/deaths against Google data was a simple and indirect way to deal with seasonality. More complex and sophisticated approaches to deal with seasonality like those employed by Franses and Paap (2004) would require much larger datasets that are currently not available. This is why we leave this issue as an avenue for further research.

We want to emphasize that at the beginning of the pandemic the reproduction number was probably above unity for many (if not all) countries, so that it is very likely that the processes generating the COVID daily data series were explosive, that is with the unit root greater than one. In such a case, the data are not integrable of any order (similarly to financial bubble processes), and the assumptions for the unit root tests are not satisfied, so that the critical values of the tests may not be valid. However, the effects on the test result strongly depend on the specific test used and the chosen alternative hypothesis (either the stationary alternative or the explosive alternative), see Haldrup and Lildholdt (2002) for details. Given this issue, we decided to take a neutral stance, and the subsequent empirical analysis considered models for data in levels, log-levels, first-differences, and log-returns. In this regard, we emphasize that a large part of the epidemiologic literature does not deal with non-stationarity at all, see Cazelles et al. (2018) and references therein for more details ${ }^{5}$.

Following Li et al. (2020b), the first step of the empirical analysis was the computation of the lag correlations between the daily COVID-19 cases for each country and Google search data for the topics 'coronavirus' and 'pneumonia' up to a 30-day horizon. The 30-day horizon was chosen based on the limited size of our dataset and on past literature, see Li et al. (2020b) and references therein. For the sake of space and interest, we report in Fig. 2 the violin plots (that is, kernel densities + box plot) of the lags showing the highest correlation between the daily COVID-19 cases and Google searches across all countries, for both variables in levels and first-differences. Instead, the highest lagged correlations together with their corresponding lags for each country are reported in the Supplementary Table S1 and S2, for the case of variables in levels and in first differences, respectively ${ }^{6}$.

Figure 2 shows that the median lag with the highest correlation across all countries is 23 days (levels) / 17 days (first differences) for the 'pneumonia' topic, while it is 20 days (levels) / 18 days (first differences) for the 'coronavirus' topic. In general, these results are fairly stable across data in levels and first differences and quite close to those reported by Li et al. (2020b), even though somewhat higher than the latter. In this regard, we remark again that the WHO (2020) states that "the time between exposure to COVID-19 and the moment when symptoms start is commonly around five to six days but can range from 1-14 days". Moreover, the fact that infected people may wait some time before contacting a doctor (fear of job loss, attempt to self-medicate, etc.), together with the objective difficulty to get tested in several countries, can explain why the lags with the highest correlation are generally higher than those reported for China by Li et al. (2020b).

5 Testing for Granger causality using the Toda-Yamamoto (1995) approach should not particularly suffer from processes that are mildly explosive and have this feature only at the beginning of the pandemic. This follows from Haldrup and Lildholdt (2002), who showed that $I(2)$ processes have properties that mimic those of explosive processes in finite samples, and from the fact that the Toda-Yamamoto approach is valid with processes which may be integrated or cointegrated of arbitrary order. Moreover, this approach has been found to be valid with a larger set of processes, as shown by the large simulation studies performed by Hacker and Hatemi (2006).

6 The supplementary materials can be found on the author's website. 


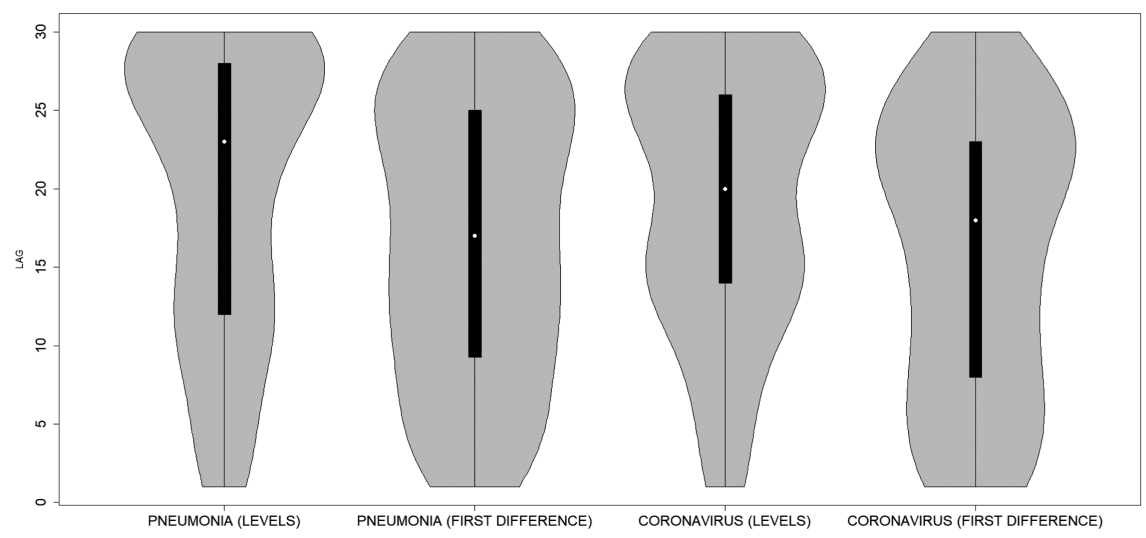

Fig. 2. Violin plots of the lags with the highest correlations between new COVID-19 cases and Google searches for the topics 'coronavirus' and 'pneumonia' across 158 countries, January-May 2020

\subsection{Granger Causality}

Even though lag-correlations are a useful tool to gain a general idea about potential predictability, it is better to compute a Granger-causality (GC) test to formally test whether one (or more) time series can be useful in forecasting another time series. The violin plots of the $p$-values for the null hypothesis that Google searches for the topics 'coronavirus' and 'pneumonia' do not Granger-cause the daily new COVID-19 cases, using vector autoregressive models with optimal lags selected with the Akaike information criteria (for both the variables in levels and in first differences), are reported in Fig. 3. The same figure also reports the $p$-values for the null hypothesis of no Granger-causality using the approach by Toda-Yamamoto, which is valid even if the processes may be integrated or cointegrated of arbitrary order.

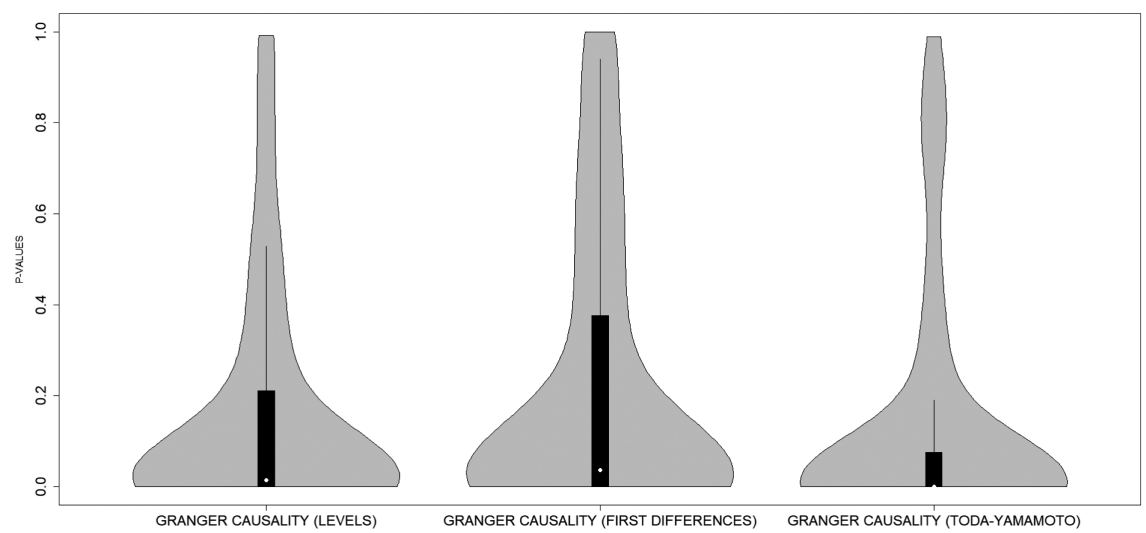

Fig. 3. Violin plots of the $p$-values for the null hypothesis that the Google searches for the topics 'coronavirus' and 'pneumonia' do not Granger-cause the daily new COVID-19 cases across 158 countries, January-May 2020 
The detailed $p$-values for the null hypothesis that the Google searches do not Granger-cause the daily new COVID-19 cases across 158 countries are reported in the Supplementary Table S3: the null hypothesis was rejected at the $5 \%$ probability level for 87 out of 158 countries using variables in levels, for 80 countries using variables in first differences, and for 106 countries using the approach by Toda and Yamamoto. Therefore, there is strong evidence that lagged Google search data do explain the variation in the daily new COVID-19 cases for the vast majority of countries worldwide. It is interesting to note that when we move to an approach like the TodaYamamoto methodology able to deal with non-stationarity (and potentially explosive processes, given that $I(2)$ processes mimic explosive processes in finite samples), the evidence in favor of Google data further increases.

\subsection{Out-of-sample forecasting}

The last step to evaluate the ability of Google search data to predict the COVID-19 outbreak was to perform an out-of-sample forecasting analysis for each country, to forecast the number of daily new cases using several competing models with and without Google data. Three classes of models were considered for a total of 18 models:

1) Time series models with the daily number of new COVID-19 cases as the dependent variable: a. ARIMA models with the variables in levels, log-levels, first differences and log-returns.

Total: 4 models.

b. Error-Trend-Seasonal (ETS) model. Total: 1 model.

2) Google-augmented time series models:

a. ARIMA-X with lagged Google data for the topic 'pneumonia' as an exogenous repressor, with the variables in levels, log-levels, first differences and log-returns. Total: 4 models.

b. VAR models with the variables in levels, log-levels, first differences and log-returns. The endogenous regressors are the daily number of new COVID-19 cases and the Google search data for the topics 'coronavirus' and 'pneumonia'. Total: 4 models.

c. The Hierarchical Vector Autoregression (HVAR) model estimated with the Least Absolute Shrinkage and Selection Operator (LASSO), with the variables in levels, log-levels, first differences and log-returns. Total: 4 models.

3) The SIR compartmental epidemic model. Total: 1 model.

Additional models could surely be added, but this selection already gave important indications whether Google search data are useful for forecasting the daily cases of COVID-19. Given the previous evidence with lag correlations and Granger-causality tests, a forecasting horizon of 14 days was considered. Note that precise forecasts over a 2-week horizon can be extremely important for policy makers and health officers.

The data in January-March 2020 were used as the first training sample for the models' estimation, while April-May 2020 was left for out-of-sample forecasting using an expanding estimation window. A summary of the models' performances across the 158 countries according to the mean squared error (MSE) and the mean absolute error (MAE) is reported in Table 1, respectively, while the top 3 models for each country are reported in the Supplementary Table S4 and Supplementary Table S5, respectively.

Google-augmented time series models were the best models for 86/89 countries out of 158 according to the MSE/MAE (mainly ARIMA-X models and HVAR models). The SIR model confirmed its good name by being the top model for almost $20 \%$ of the countries examined. 
Table 1. Summary of the models' performances across the 158 countries (according to the MSE and the MAE) for the out-of-sample period in April-May 2020

\begin{tabular}{llcccccc}
\hline & \multicolumn{6}{c}{ Number of times the model was } \\
\cline { 2 - 8 } & \multicolumn{1}{c}{ MSE } & \multicolumn{4}{c}{ MAE } \\
\hline Time series models & $1^{\text {st }}$ best & $2^{\text {nd }}$ best & $3^{\text {rd }}$ best & $1^{\text {st }}$ best & $2^{\text {nd }}$ best & $3^{\text {rd }}$ best \\
& ARIMA & 7 & 9 & 21 & 5 & 17 & 16 \\
& ARIMA.LOG & 16 & 15 & 7 & 20 & 17 & 18 \\
& ARIMA.DIFF & 11 & 21 & 22 & 9 & 16 & 16 \\
& ARIMA.DIFFLOG & 2 & 4 & 3 & 0 & 3 & 5 \\
Google-augmented & ETS & 6 & 6 & 12 & 7 & 14 & 11 \\
time series models & ARIMAX & 9 & 13 & 17 & 7 & 11 & 17 \\
& ARIMAX.LOG & 12 & 14 & 10 & 12 & 19 & 13 \\
& ARIMAX.DIFF & 15 & 24 & 16 & 18 & 15 & 12 \\
& ARIMAX.DIFFLOG & 13 & 3 & 7 & 8 & 3 & 7 \\
& VAR & 0 & 0 & 0 & 0 & 0 & 0 \\
& VAR.LOG & 1 & 0 & 1 & 1 & 0 & 1 \\
& VAR.DIFF & 0 & 0 & 0 & 0 & 0 & 0 \\
& VAR.DLOG & 0 & 2 & 4 & 0 & 3 & 4 \\
& HVAR & 15 & 5 & 6 & 11 & 5 & 7 \\
& HVAR.LOG & 10 & 12 & 6 & 19 & 8 & 9 \\
& HVAR.DIFF & 7 & 11 & 8 & 8 & 5 & 11 \\
Compartmental epidemic & HVAR.DLOG & 4 & 5 & 8 & 5 & 8 & 8 \\
& SIR & 30 & 14 & 10 & 28 & 14 & 3 \\
\hline
\end{tabular}

There are no major differences between models with the variables in levels and in first-differences, whereas models in log-levels seem to show slightly better performances across different models' specifications. VAR models performed very poorly and in several occasions did not reach numerical convergence, due to the large number of parameters involved with small sample sizes.

The previous loss functions were then used with the Model Confidence Set (MCS) by Hansen et al. (2011) to select the best forecasting models at a specified confidence level. Once the loss differential between models $i$ and $j$ at time $t$ are computed (that is $d_{i, j, t}=L_{i, t}-L_{j, t}$ ), the MCS approach tests the hypothesis of equal predictive ability, $\mathrm{H}_{0, M}: \mathrm{E}\left(d_{i, j, t}\right)=0$, for all $i, j \in M$, where $M$ is the set of forecasting models. First, the following $t$-statistics are computed, $t_{i \bullet}=\bar{d}_{i \bullet} / \widehat{\operatorname{var}}\left(\bar{d}_{i \bullet}\right)$ for $i \in M$, where $\bar{d}_{i \bullet}=m^{-1} \sum_{j \in M} \bar{d}_{i j}$ is the simple loss of the $i$-th model relative to the average losses across models in the set $M, \bar{d}_{i j}=T^{-1} \sum_{t=1}^{T} d_{i j, t}$ measures the sample loss differential between model $i$ and $j$, and $\widehat{\operatorname{var}}\left(\bar{d}_{i}\right)$ is an estimate of $\operatorname{var}\left(\bar{d}_{i}\right)$. Secondly, the following test statistic is computed to test for the null hypothesis: $T_{\max }=\max _{i \in M}\left(t_{i}\right)$. This statistic has a non-standard distribution, so the distribution under the null hypothesis is computed using bootstrap methods with 2000 replications. If the null hypothesis is rejected, one model is eliminated from the analysis and the testing procedure starts again.

The number of times each model was included into the MCS across the 158 countries, according to the MSE and the MAE, is reported in Table 2. 
Table 2. Number of times each model was included into the MCS across the 158 countries

\begin{tabular}{ll|ll}
\hline \multicolumn{1}{c}{ Loss: MSE } & Loss: MAE \\
\hline ARIMA & 145 & ARIMA & 127 \\
ARIMA.LOG & 147 & ARIMA.LOG & 131 \\
ARIMA.DIFF & 145 & ARIMA.DIFF & 133 \\
ARIMAX.DIFFLOG & 130 & ARIMAX.DIFFLOG & 116 \\
ETS & 142 & ETS & 122 \\
ARIMAX & 144 & ARIMAX & 125 \\
ARIMAX.LOG & 146 & ARIMAX.LOG & 130 \\
ARIMAX.DIFF & 141 & ARIMAX.DIFF & 129 \\
ARIMA.DIFFLOG & 133 & ARIMA.DIFFLOG & 115 \\
VAR & 96 & VAR & 76 \\
VAR.LOG & 74 & VAR.LOG & 59 \\
VAR.DIFF & 99 & VAR.DIFF & 75 \\
VAR.DLOG & 102 & VAR.DLOG & 90 \\
HVAR & 138 & HVAR & 115 \\
HVAR.LOG & 138 & HVAR.LOG & 127 \\
HVAR.DIFF & 138 & HVAR.DIFF & 121 \\
HVAR.DLOG & 134 & HVAR.DLOG & 116 \\
SIR & 138 & SIR & 116 \\
\hline
\end{tabular}

With the exception of VAR models, almost all models were included in the MCS, thus showing that there was not enough information in the data to partition good and bad models: this outcome was expected given the small sample used in this forecasting exercise.

\section{Robustness check: Modelling and forecasting the number of COVID-19 deaths}

A common critique to the analysis of COVID-19 cases is that the number of cases could depend on the amount of testes, so that a country could have few official cases due to low testing. Therefore, modelling and forecasting the number of COVID-19 deaths could be a better metric to evaluate the forecasting ability of Google search data. We repeated the previous analysis with the daily deaths for COVID-19, considering only those countries with at least 100 deaths (for a total of 64 countries). The violin plots of the lags with the highest correlations between new daily COVID-19 deaths and Google searches for the topics 'coronavirus' and 'pneumonia' is reported in Fig. 4. The violin plots of the $p$-values for the null hypothesis that the Google searches for the topics 'coronavirus' and 'pneumonia' do not Granger-cause the daily COVID-19 deaths across 158 countries is reported in Fig. 5.

Figure 4 shows that the median lag with the highest correlation across all countries is 28 days (levels) / 19 days (first differences) for the 'pneumonia' topic, while it is 26 days (levels) / 18 days (first differences) for the 'coronavirus' topic: the highest correlations in levels take place approximately 1 week later that those for the confirmed cases, which makes sense from an epidemiologic point of view.

The null hypothesis that the Google searches do not Granger-cause the daily new COVID-19 deaths across 64 countries was rejected at the 5\% probability level for 40 countries using variables in levels, for 30 countries using variables in first differences, and for 42 countries using the approach by Toda and Yamamoto. Therefore, there is strong evidence that lagged Google search data do explain the variation in the daily new COVID-19 deaths for most of the countries examined. 


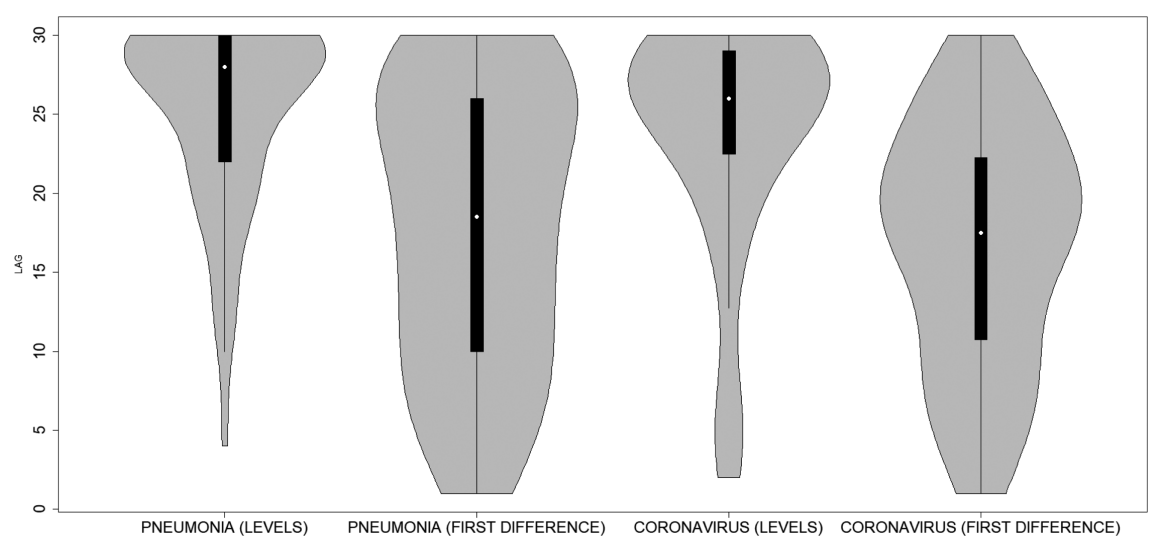

Fig. 4. Violin plots of the lags with the highest correlations between daily COVID-19 deaths and Google searches for the topics 'coronavirus' and 'pneumonia' across 158 countries, January-May 2020

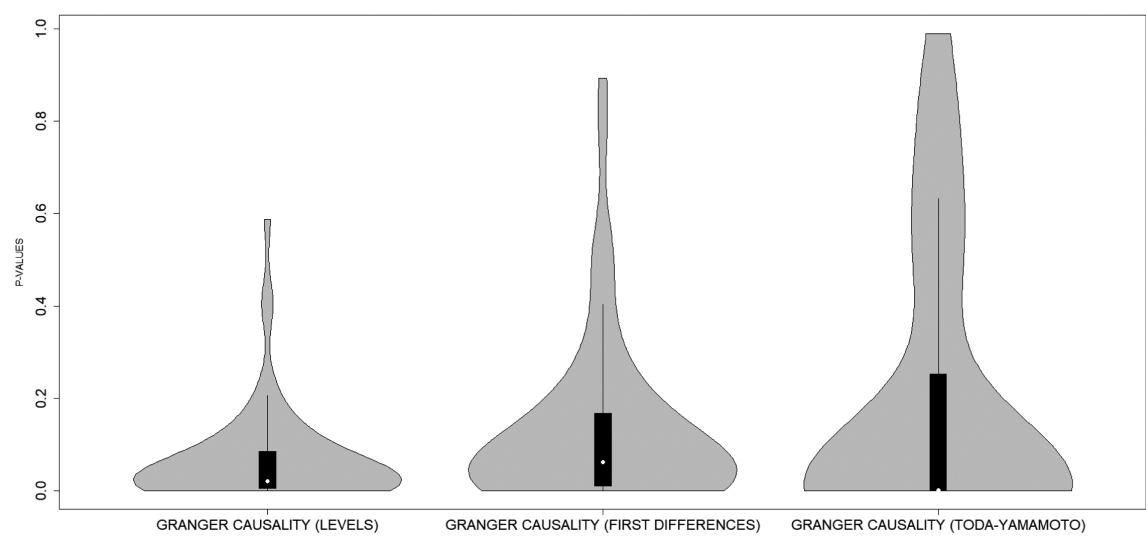

Fig. 5. Violin plots of the $p$-values for the null hypothesis that the Google searches for the topics 'coronavirus' and 'pneumonia' do not Granger-cause the daily COVID-19 deaths across 158 countries, January-May 2020

The out-of-sample forecasting analysis involved again the previous 17 time series models, but they used the daily number of new COVID-19 deaths as the dependent variable, instead. As for epidemiologic models, we considered two variants:

a) the SIR model described by the system of equations (2) where the number of infected is substituted with the number of deaths, thus implying that the mortality rate for those infected is $100 \%$. This is clearly a biased (and unrealistic) model, but it has a benefit to be numerically more efficient than more complex epidemic models. Moreover, it can be interpreted as a shrinkage estimator, see Lehmann and Casella (1998);

b) the SIRD model described by the system of equations (3), which is a benchmark model in epidemiology when both people infected and deaths have to be modelled.

A summary of the models' performances across the 64 countries according to the mean squared error (MSE) and the mean absolute error (MAE) is reported in Table 3. 
Table 3. Summary of the models' performances when forecasting the daily deaths for COVID-19 across the 64 countries (according to the MSE and the MAE) for the out-of-sample period in April-May 2020

\begin{tabular}{|c|c|c|c|c|c|c|c|}
\hline & \multirow[b]{2}{*}{ Models } & \multicolumn{3}{|c|}{ MSE } & \multicolumn{3}{|c|}{ MAE } \\
\hline & & $1^{\text {st }}$ best & $2^{\text {nd }}$ best & $3^{\text {rd }}$ best & $1^{\text {st }}$ best & $2^{\text {nd }}$ best & $3^{\text {rd }}$ best \\
\hline \multirow[t]{9}{*}{ Time series models } & ARIMA & 5 & 11 & 6 & 2 & 9 & 8 \\
\hline & ARIMA.LOG & 1 & 2 & 3 & 2 & 8 & 6 \\
\hline & ARIMA.DIFF & 5 & 7 & 7 & 7 & 6 & 8 \\
\hline & ARIMA.DIFFLOG & 2 & 7 & 3 & 1 & 5 & 4 \\
\hline & ETS & 2 & 0 & 0 & 1 & 2 & 2 \\
\hline & ARIMAX & 3 & 5 & 8 & 8 & 3 & 3 \\
\hline & ARIMAX.LOG & 1 & 1 & 4 & 2 & 5 & 5 \\
\hline & ARIMAX.DIFF & 17 & 7 & 5 & 13 & 10 & 3 \\
\hline & ARIMAX.DIFFLOG & 4 & 4 & 2 & 5 & 1 & 1 \\
\hline \multirow{8}{*}{$\begin{array}{l}\text { Google-augmented } \\
\text { time series models }\end{array}$} & VAR & 0 & 0 & 0 & 0 & 0 & 0 \\
\hline & VAR.LOG & 0 & 0 & 0 & 0 & 0 & 1 \\
\hline & VAR.DIFF & 0 & 0 & 0 & 0 & 0 & 0 \\
\hline & VAR.DLOG & 1 & 0 & 1 & 0 & 1 & 1 \\
\hline & HVAR & 5 & 2 & 4 & 6 & 4 & 2 \\
\hline & HVAR.LOG & 1 & 2 & 0 & 1 & 1 & 1 \\
\hline & HVAR.DIFF & 4 & 4 & 6 & 1 & 5 & 7 \\
\hline & HVAR.DLOG & 2 & 4 & 6 & 3 & 2 & 5 \\
\hline \multirow[t]{2}{*}{ Compartmental epidemic model } & SIR & 8 & 7 & 9 & 11 & 2 & 7 \\
\hline & SIRD & 3 & 1 & 0 & 1 & 0 & 0 \\
\hline
\end{tabular}

Google-augmented time series models were the best models for 38/39 countries out of 64 according to the MSE/MAE (again ARIMA-X models and HVAR models). There is no major differences between models with the variables in levels and in first-differences, but ARIMA-X models in first differences seem to show better performances than competing models. VAR models performed again very poorly. The SIR and SIRD model were the top models for approximately $18 \%$ of the countries examined: interestingly, the SIR model performed better than the SIRD model, thus confirming again that in some cases efficiency is more important than unbiasedness.

The number of times each model was included into the MCS across the 64 countries according to the MSE and the MAE - is reported in Table 4.

Similarly to the baseline case, almost all models were included in the MCS (with the exception of VAR models, and the SIRD model to a lower degree), thus showing again that there was not enough information in the data to separate good and bad models.

\section{Conclusions}

Google Trends data for the topics 'coronavirus' and 'pneumonia' filtered using the 'Health' category proved to be strongly predictive for the number of new daily cases and deaths of COVID-19 for a large set of countries. Google data can complement epidemiological models during difficult times like the ongoing COVID-19 pandemic, when official statistics maybe not fully reliable and/or published with a delay. Policymakers and health officials can use web searches to verify how the pandemic is developing, and/or to check the effect of their policy actions to contain the disease and to modify them if these policies prove to be unsatisfactory. 
Table 4. Number of times each model was included into the MCS across the 64 countries

\begin{tabular}{ll|ll}
\hline \multicolumn{2}{c}{ Loss: MSE } & Loss: MAE \\
\hline ARIMA & 61 & ARIMA & 58 \\
ARIMA.LOG & 58 & ARIMA.LOG & 52 \\
ARIMA.DIFF & 61 & ARIMA.DIFF & 57 \\
ARIMA.DIFFLOG & 53 & ARIMA.DIFFLOG & 54 \\
ETS & 54 & ETS & 48 \\
ARIMAX & 59 & ARIMAX & 54 \\
ARIMAX.LOG & 57 & ARIMAX.LOG & 51 \\
ARIMAX.DIFF & 60 & ARIMAX.DIFF & 59 \\
ARIMAX.DIFFLOG & 47 & ARIMAX.DIFFLOG & 44 \\
VAR & 21 & VAR & 19 \\
VAR.LOG & 16 & VAR.LOG & 20 \\
VAR.DIFF & 25 & VAR.DIFF & 22 \\
VAR.DLOG & 22 & VAR.DLOG & 26 \\
HVAR & 54 & HVAR & 50 \\
HVAR.LOG & 53 & HVAR.LOG & 42 \\
HVAR.DIFF & 58 & HVAR.DIFF & 56 \\
HVAR.DLOG & 58 & HVAR.DLOG & 53 \\
SIR & 59 & SIR & 52 \\
SIRD & 48 & SIRD & 35 \\
\hline
\end{tabular}

This is particularly important now that several countries have decided to reopen their economies, so that real-time tracking with online-data is one of the instruments that can be used to keep the situation under control.

Even though some models performed better than others did, these forecasting differences were not statistically significant due to the small samples used. An avenue of future research would be to consider larger samples and forecast combination methods, following the ideas discussed by Clemen (1989), Timmermann (2006), Hsiao and Wan (2014), and Hyndman and Athanasopoulos (2018).

Acknowledgments. The author would like to thank all the participants of the online scientific seminars on the topic "Economic Challenges of COVID-19 Pandemic" and "Applied statistics and modeling of real processes" for helpful comments and suggestions. The author gratefully acknowledges financial support from the grant of the Russian Science Foundation no. 20-68-47030.

\section{References}

Alamo T., Reina D. G., Mammarella M., Abella A. (2020). Covid-19: Open-data resources for monitoring, modeling, and forecasting the epidemic. Electronics, 9 (5), 827.

Anastassopoulou C., Russo L., Tsakris A., Siettos C. (2020). Data-based analysis, modelling and forecasting of the COVID-19 outbreak. PloS One, 15 (3), e0230405.

Ayers J. W., Althouse B. M., Allem J. P., Rosenquist J. N., Ford D. E. (2013). Seasonality in seeking mental health information on Google. American Journal of Preventive Medicine, 44 (5), 520-525.

Ayyoubzadeh S. M., Ayyoubzadeh S. M., Zahedi H., Ahmadi M., Kalhori S. R. N. (2020). Predicting COVID-19 incidence through analysis of Google Trends data in Iran: Data mining and deep learning pilot study. JMIR Public Health and Surveillance, 6 (2), e18828. 
Birrell P. J., Ketsetzis G., Gay N. J., Cooper B. S., Presanis A. M., Harris R. J., Charlett A., Zhang X. S., White P., Pebody R., De Angelis D. (2011). Bayesian modeling to unmask and predict influenza A/H1N1p$\mathrm{dm}$ dynamics in London. Proceedings of the National Academy of Sciences, 108 (45), 18238-18243.

Boyle J. R., Sparks R. S., Keijzers G. B., Crilly J. L., Lind J. F., Ryan L. M. (2011). Prediction and surveillance of influenza epidemics. Medical Journal of Australia, 194, S28-S33.

Brauer F., Castillo-Chavez C., Castillo-Chavez C. (2012). Mathematical models in population biology and epidemiology. New York: Springer.

Broniatowski D. A., Paul M. J., Dredze M. (2013). National and local influenza surveillance through Twitter: An analysis of the 2012-2013 influenza epidemic. PloS One, 8 (12), e83672.

Cazelles B., Champagne C., Dureau J. (2018). Accounting for non-stationarity in epidemiology by embedding time-varying parameters in stochastic models. PLoS Computational Biology, 14 (8), e1006211.

Clemen R. T. (1989). Combining forecasts: A review and annotated bibliography. International Journal of Forecasting, 5 (4), 559-583.

D’Amuri F., Marcucci J. (2017). The predictive power of Google searches in forecasting US unemployment. International Journal of Forecasting, 33 (4), 801-816.

Dugas A. F., Jalalpour M., Gel Y., Levin S., Torcaso F., Igusa T., Rothman R. E. (2013). Influenza forecasting with Google Flu Trends. PloS One, 8 (2), e56176.

Eichenbaum M. S., Rebelo S., Trabandt M. (2020a). The macroeconomics of epidemics. NBER Working Paper 26882. National Bureau of Economic Research.

Eichenbaum M. S., Rebelo S., Trabandt M. (2020b). The macroeconomics of testing and quarantining. NBER Working Paper 27104. National Bureau of Economic Research.

Eichenbaum M. S., Rebelo S., Trabandt M. (2020c). Epidemics in the neoclassical and New Keynesian models. NBER Working Paper 27430. National Bureau of Economic Research.

European Centre for Disease Prevention and Control (ECDC, 2020). Download today's data on the geographic distribution of COVID-19 cases worldwide. https://www.ecdc.europa.eu/en/publications-data/ download-todays-data-geographic-distribution-covid-19-cases-worldwide.

Fantazzini D. (2014). Nowcasting and forecasting the monthly food stamps data in the US using online search data. PloS One, 9 (11), e111894.

Fantazzini D. (2019). Quantitative finance with $R$ and cryptocurrencies. Amazon KDP, ISBN-13, 9781090685315.

Fantazzini D., Toktamysova Z. (2015). Forecasting German car sales using Google data and multivariate models. International Journal of Production Economics, 170, 97-135.

Franses P. H., Paap R. (2004). Periodic time series models. OUP Oxford.

Gencoglu O., Gruber M. (2020). Causal modeling of twitter activity during COVID-19. arXiv preprint, arXiv:2005.07952.

Gianfredi V., Bragazzi N. L., Mahamid M., Bisharat B., Mahroum N., Amital H., Adawi M. (2018). Monitoring public interest toward pertussis outbreaks: An extensive Google Trends-based analysis. Public Health, 165, 9-15.

Ginsberg J., Mohebbi M. H., Patel R. S., Brammer L., Smolinski M. S., Brilliant L. (2009). Detecting influenza epidemics using search engine query data. Nature, 457 (7232), 1012-1014.

Granger C. W. (1969). Investigating causal relations by econometric models and cross-spectral methods. Econometrica, 424-438. 
Granger C. W. (1980). Testing for causality: A personal viewpoint. Journal of Economic Dynamics and control, 2, 329-352.

Granger C. W., Newbold P. (1974). Spurious regressions in econometrics. Journal of Econometrics, 2, 111-120.

Hacker R. S., Hatemi J. A. (2006). Tests for causality between integrated variables using asymptotic and bootstrap distributions: Theory and application. Applied Economics, 38 (13), 1489-1500.

Haldrup N., Lildholdt P. (2002). On the robustness of unit root tests in the presence of double unit roots. Journal of Time Series Analysis, 23 (2), 155-171.

Hall I. M., Gani R., Hughes H. E., Leach S. (2007). Real-time epidemic forecasting for pandemic influenza. Epidemiology and Infection, 135 (3), 372-385.

Hansen P. R., Lunde A., Nason J. M. (2011). The model confidence set. Econometrica, 79 (2), 453-497.

Ho H. T., Carvajal T. M., Bautista J. R., Capistrano J. D. R., Viacrusis K. M., Hernandez L. F. T., Watanabe K. (2018). Using Google Trends to examine the spatio-temporal incidence and behavioral patterns of dengue disease: A case study in Metropolitan Manila, Philippines. Tropical Medicine and Infectious Disease, 3 (4), 118.

Hsiao C., Wan S. K. (2014). Is there an optimal forecast combination? Journal of Econometrics, 178, 294-309.

Hyndman R. J., Koehler A. B., Snyder R. D., Grose S. (2002). A state space framework for automatic forecasting using exponential smoothing methods. International Journal of Forecasting, 18 (3), 439-454.

Hyndman R., Koehler A. B., Ord J. K., Snyder R. D. (2008). Forecasting with exponential smoothing: The state space approach. Springer Science and Business Media.

Hyndman R. J., Athanasopoulos G. (2018). Forecasting: Principles and practice. OTexts.

Kermack W. O., McKendrick A. G. (1927). A contribution to the mathematical theory of epidemics. Proceedings of the Royal Society of London. Series A, 115 (772), 700-721.

Lazer D., Kennedy R., King G., Vespignani A. (2014). The parable of Google Flu: Traps in big data analysis. Science, 343 (6176), 1203-1205.

Lehmann E. L., Casella G. (1998). Theory of point estimation. Springer Science and Business Media.

Li Q., Guan X., Wu P., Wang X., Zhou L., Tong Y., Ren R., Leung K. S., Lau E. H., Wong J. Y., Xing X. (2020a). Early transmission dynamics in Wuhan, China, of novel coronavirus-infected pneumonia. New England Journal of Medicine, 382 (13), 1199-1207.

Li C., Chen L. J., Chen X., Zhang M., Pang C. P., Chen H. (2020b). Retrospective analysis of the possibility of predicting the COVID-19 outbreak from Internet searches and social media data, China, 2020. Eurosurveillance, 25 (10), 2000199.

Longini I. M., Fine P. E., Thacker S. B. (1986). Predicting the Global Spread of new infectious agents. American Journal of Epidemiology, 123 (3), 383-391.

Lütkepohl H. (2005). New introduction to multiple time series analysis. Springer Science and Business Media.

Makridakis S., Hibon M. (2000). The M3-Competition: Results, conclusions and implications. International Journal of Forecasting, 16 (4), 451-476.

Makridakis S., Spiliotis E., Assimakopoulos V. (2020). The M4 Competition: 100,000 time series and 61 forecasting methods. International Journal of Forecasting, 36 (1), 54-74.

Majumder M. S., Santillana M., Mekaru S. R., McGinnis D. P., Khan K., Brownstein J. S. (2016). Utilizing nontraditional data sources for near real-time estimation of transmission dynamics during the 2015-2016 Colombian Zika virus disease outbreak. JMIR Public Health and Surveillance, 2 (1), e30. 
Milinovich G. J., Williams G. M., Clements A. C., Hu W. (2014). Internet-based surveillance systems for monitoring emerging infectious diseases. The Lancet Infectious Diseases, 14 (2), 160-168.

Marques-Toledo C., Degener C. M., Vinhal L., Coelho G., Meira W., Codeço C. T., Teixeira M. M. (2017). Dengue prediction by the web: Tweets are a useful tool for estimating and forecasting Dengue at country and city level. PLoS Neglected Tropical Diseases, 11 (7), e0005729.

Nicholson W. B., Matteson D. S., Bien J. (2017). VARX-L: Structured regularization for large vector autoregressions with exogenous variables. International Journal of Forecasting, 33 (3), 627-651.

Nicholson W. B., Wilms I., Bien J., Matteson D. S. (2018). High dimensional forecasting via interpretable vector autoregression. arXiv preprint, arXiv:1412.5250.

Nicholson W., Matteson D., Bien J. (2019). BigVAR: Dimension reduction methods for multivariate time series. R package version 1.0.6. https://cran.r-project.org/web/packages/BigVAR/BigVAR.pdf.

Park J. Y., Phillips P. C. (1989). Statistical inference in regressions with integrated processes: Part 2. Econometric Theory, 5 (1), 95-131.

Phillips P. C. (1986). Understanding spurious regressions in econometrics. Journal of Econometrics, 33 (3), 311-340.

Santangelo O. E., Provenzano S., Piazza D., Giordano D., Calamusa G., Firenze A. (2019). Digital epidemiology: Assessment of measles infection through Google Trends mechanism in Italy. Annali di Igiene, 31, 385-391.

Santillana M., Nguyen A. T., Dredze M., Paul M. J., Nsoesie E. O., Brownstein J. S. (2015). Combining search, social media, and traditional data sources to improve influenza surveillance. PLoS Computational Biology, 11 (10).

Seifter A., Schwarzwalder A., Geis K., Aucott J. (2010). The utility of "Google Trends" for epidemiological research: Lyme disease as an example. Geospatial Health, 4, 135-137.

Shin S. Y., Seo D. W., An J., Kwak H., Kim S. H., Gwack J., Jo M. W. (2016). High correlation of Middle East respiratory syndrome spread with Google search and Twitter trends in Korea. Scientific Reports, 6, 32920 .

Shinde G. R., Kalamkar A. B., Mahalle P. N., Dey N., Chaki J., Hassanien A. E. (2020). Forecasting models for coronavirus disease (COVID-19): A survey of the state-of-the-art. SN Computer Science, 1 (4), 1-15.

Sims C. A., Stock J. H., Watson M. W. (1990). Inference in linear time series models with some unit roots. Econometrica, 58, 113-144.

Soetaert K., Petzoldt T., Setzer R. W. (2010a). Solving differential equations in R. The R Journal, 2 (2), $5-15$.

Soetaert K. E., Petzoldt T., Setzer, R. W. (2010b). Solving differential equations in R: Package deSolve. Journal of Statistical Software, 33, 1-25.

Stock J., Watson M. (1989). Interpreting the evidence on money-income causality. Journal of Econometrics, 40 (1), 161-182.

Timmermann A. (2006). Forecast combinations. Handbook of Economic Forecasting, 1, 135-196.

Teng Y., Bi D., Xie G., Jin Y., Huang Y., Lin B., An X., Feng D., Tong Y. (2017). Dynamic forecasting of Zika epidemics using Google Trends. PloS one, 12 (1), e0165085.

Toda H. Y., Yamamoto T. (1995). Statistical inference in vector autoregressions with possibly integrated processes. Journal of Econometrics, 66 (1-2), 225-250.

Valdivia A., Monge-Corella S. (2010). Diseases tracked by using Google Trends, Spain. Emerging Infectious Diseases, 16 (1), 168-169. 
Yang S., Santillana M., Kou S. C. (2015). Accurate estimation of influenza epidemics using Google search data via ARGO. Proceedings of the National Academy of Sciences, 112 (47), 14473-14478.

Yin S., Ho M. (2012). Monitoring a toxicological outbreak using Internet search query data. Clinical Toxicology, 50 (9), 818-822.

Yuan Q., Nsoesie E. O., Lv B., Peng G., Chunara R., Brownstein J. S. (2013). Monitoring influenza epidemics in China with search query from Baidu. PLoS One, 8, e64323.

Wiener N. (1956). The theory of prediction. In: Beckenbach E. (ed.), Modern Mathematics for Engineers. McGraw-Hill, New York, 165-190.

Wilson K., Brownstein J. S. (2009). Early detection of disease outbreaks using the Internet. Canadian Medical Association Journal, 180 (8), 829-831.

WHO (2020). WHO characterizes COVID-19 as a pandemic. https://www.who.int/emergencies/diseases/ novel-coronavirus-2019/events-as-they-happen.

Zhou X., Ye J., Feng Y. (2011). Tuberculosis surveillance by analyzing Google Trends. IEEE Transactions on Biomedical Engineering, 58 (8), 2247-2254.

Received 12.06.2020; accepted 10.08.2020.

\section{Appendix A: Google Trends}

Google Trends is a website (https://trends.google.com) that reports the standardized volume of Google searches for a keyword or a topic. The data can be filtered according to search type (for example, web, videos, images), search category (there are 25 categories and 288 subcategories), geographic location, and time range.

Google Trends calculates the ratio of the number of online searches for a specific keyword/ topic $K$ in a given geographical region $a$ on a particular day $t\left(K_{a, t}\right)$, to the total amount of searches for the same day and region $\left(T_{a, t}\right): R_{a, t}=\frac{K_{a, t}}{T_{a, t}}$.

The resulting time series is divided by the value of the day in which it reaches the maximum level, and multiplied by 100 . Therefore, the Google index (GI) for a specific keyword/topic $K$, on day $t$, and in area $a$ is given by, $G I_{K, a, t}=\frac{100}{\max _{t}\left(R_{a, t}\right)} R_{a, t}$.

We remark that Google Trends data are computed using a sampling method, so the results can slightly differ if the data are downloaded on different days. A possible way to decrease the sample variability is to compute the GIs as the simple average of different data downloads, carried out over different days and/or with different IPs. However, given the large dataset involved dealing with almost 200 countries and several topics, we decided to use the raw data coming from the single downloads: similarly to Fantazzini and Toktamysova (2015) and D'Amuri and Marcucci (2017), we found that using the raw data does not alter the final results, because the elementary time series are nearly identical, with cross-correlations that are never below 0.99 . 
Google Trends tracks only queries with a minimum volume due to privacy considerations: if the search volume is insufficient, a value of zero is reported ${ }^{7}$. The data are available from an intraday time-frequency up to a monthly frequency, depending on the selected time range. Google Trends allows comparing the search volumes of up to five search terms, or up to a maximum of 30 search terms grouped in a single entry using quotation marks (to return searches that match an exact expression), and using the "+" or "-" signs between the search terms to include or exclude search terms, respectively. The data are available since 2004, for several countries and their regions, see https://support.google.com/trends for more details.

Finally, it is important to note that the longer is the time sample selected, the lower is the frequency provided by Google Trends (the lowest frequency possible is monthly data). Given that we downloaded the data for only 5 months (from January 2020 till May 2020), our data consists of daily GIs. However, we want to remark that long datasets of high-frequency data can be easily obtained by chaining together several small samples of high-frequency data using a longer sample of low-frequency data. An example of the Google Trends interface to download the daily data for the topic of "Pneumonia" searched in Russia from the 01/01/2020 until the 13/05/2020, and filtered with the Health category is reported in Fig. A1.

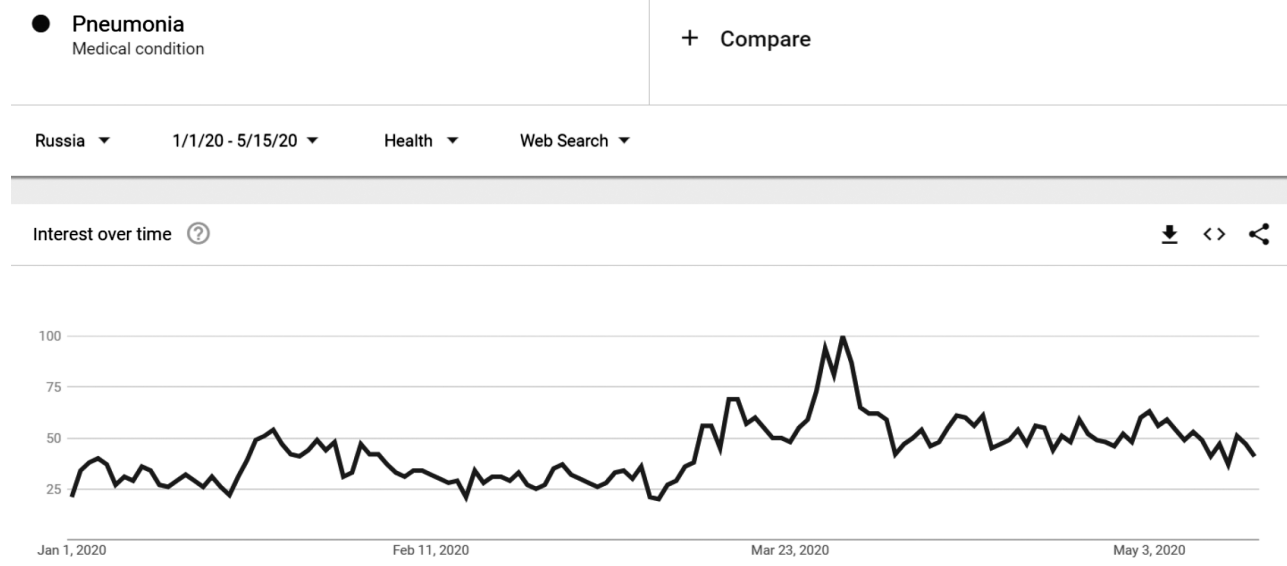

Fig. A1. Google Trends data for the topic "Pneumonia", searched in Russia, filtered using the Health category. Sample: 01/01/2020-13/05/2020

Of course, the manual download of the GIs for all countries and topics would have been too cumbersome, so that it was executed using an $\mathrm{R}$ script and the gtrends $R$ package.

7 In the case of zero values, the GIs were linearly re-scaled using a small positive constant, following the approach proposed by Fantazzini and Toktamysova (2015). The same method was employed in the case of zero values for COVID-19 cases. 